\title{
Features of the structure of seismically active zones earthquakes by satellite measurements Grace
}

\author{
Valentine B. Kashkin*a, Konstantin V. Simonov ${ }^{\mathrm{b}}$, Tatyana V. Rubleva ${ }^{\mathrm{a}}$, Alexander N. Matsulev ${ }^{\mathrm{b}}$

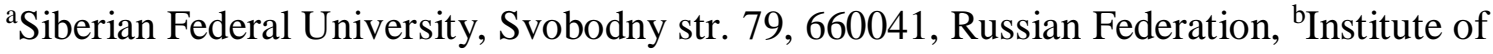 \\ Computational Modeling of the Siberian Branch of the Russian Academy of Sciences, \\ Akademgorodok 50/44, 660036 Krasnoyarsk, Russian Federation
}

\begin{abstract}
The study is devoted to the analysis of the features of the EWH (Equivalent Water Height) parameter over the geoid using satellite measurements of the GRACE space system in seismically active areas of the strongest underwater earthquakes. Created archive of satellite data GRACE. Interpretation of the disturbed state of the geomedia using digital maps of the spatial distribution of the parameter EWH is performed.
\end{abstract}

Keywords: Gravitational field, geoid, parameter EWH, underwater earthquakes, space system GRACE, satellite data processing, gravitational anomaly, seismic activity

\section{INTRODUCTION}

Satellite measurements of the GRACE CS of the height of the water surface relative to the geoid contour allow determining the deviation of the free surface of the sea from its average level along the satellite path [1]. A geoid is an equipotential surface of the field of gravity, which corresponds to the average level of the waters of the World Ocean at rest and balance, conditionally continued under the continents. The redistribution of water in the hydrosphere occurs in a thin layer near the surface of the Earth just a few kilometers wide. The monthly data obtained from the site [2] contains information on the deviation of the average ocean level from the geoid in units of measurement of the equivalent water level of EWH (Equivalent Water Height).

Basically, the EWH parameter is used to analyze the dynamic topography of the oceans and refine the parameters of the global gravitational field of the Earth [3]. EWH is calculated on the basis of the harmonic coefficients of geopotential models minus the coefficients of the average model (EIGEN-6C) [3]. This is a global model, the initial data of which are ground-based gravimetric measurements, laser trajectory observations of LAGEOS satellites (1985-2010), as well as satellite information of the GOCE and GRACE satellite stations. The equivalent water level (parameter EWH) is found from the ratio of surface density $\Delta \sigma$ to average water density $\rho_{\mathrm{w}}$ : EWH $=\Delta \sigma / \rho_{\mathrm{w}}$, where the average water density $\rho \mathrm{w}$ is $1000 \mathrm{~kg} / \mathrm{m}^{3}[4]$.

\section{FORMATTING OF MANUSCRIPT COMPONENTS}

On the basis of the satellite data GRACE [2], digital maps and three-dimensional images of the space-time distribution of the parameter EWH with a period of 30 days were constructed. An example of a digital map for the Sumatra earthquake region for December 2004 is presented in Figure 1, a. In fig. 1, b shows the image of the focal region of this earthquake in the form of a 3D model for February 2005. It should be emphasized that the images in Figure 1 reflect the structural features of the underlying perturbed geomedia.

*rtcvbk@rambler.ru 


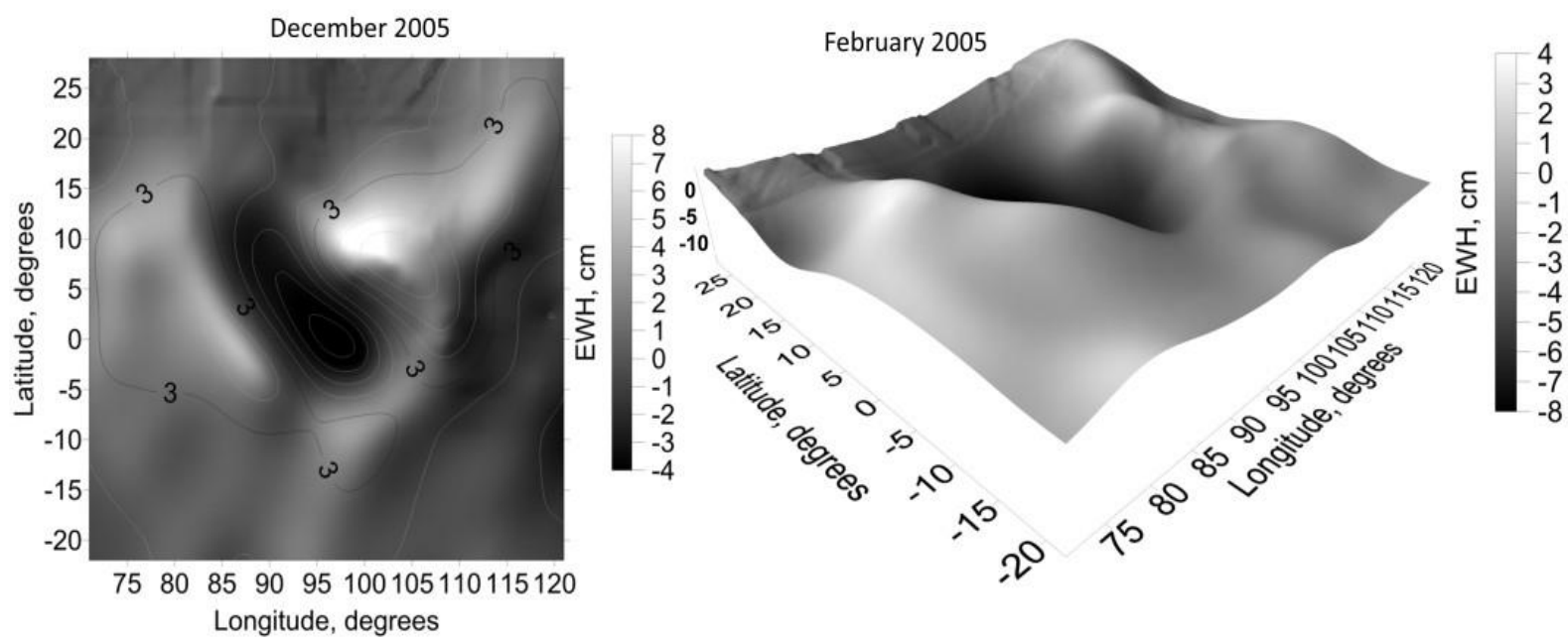

Figure 1. Digital map in contours for December 2004 (a) and 3D models for February 2005 (b) distribution of the EWH parameter of the Sumatra earthquake.

In this work, the focal region of a strong earthquake that occurred off the coast of Chile on February 27, 2010 was considered in more detail. According to the US Geological Survey, the earthquake had a magnitude Mw $=8.8$ with coordinates of a hypocenter of $35.91^{\circ} \mathrm{S}, 72.73^{\circ} \mathrm{W}$ and a depth of $35 \mathrm{~km}[5]$.

Note that the study area is characterized by frequent underwater earthquakes. Figure 2 shows a digital map of the spatial distribution of EWH for a seismically active region of Chile for February 2010 during the period of preparation and occurrence of the expected strongest earthquake, constructed using satellite data GRACE. It was revealed that the hypocenter of the earthquake (point in Fig. 2) is located near the axis of the anomaly, which is oriented submeridionally, on the maximum gradients of the parameter EWH. The interpretation of the directivity of the identified anomaly is related to the geometry of the subduction zone of lithospheric plates.

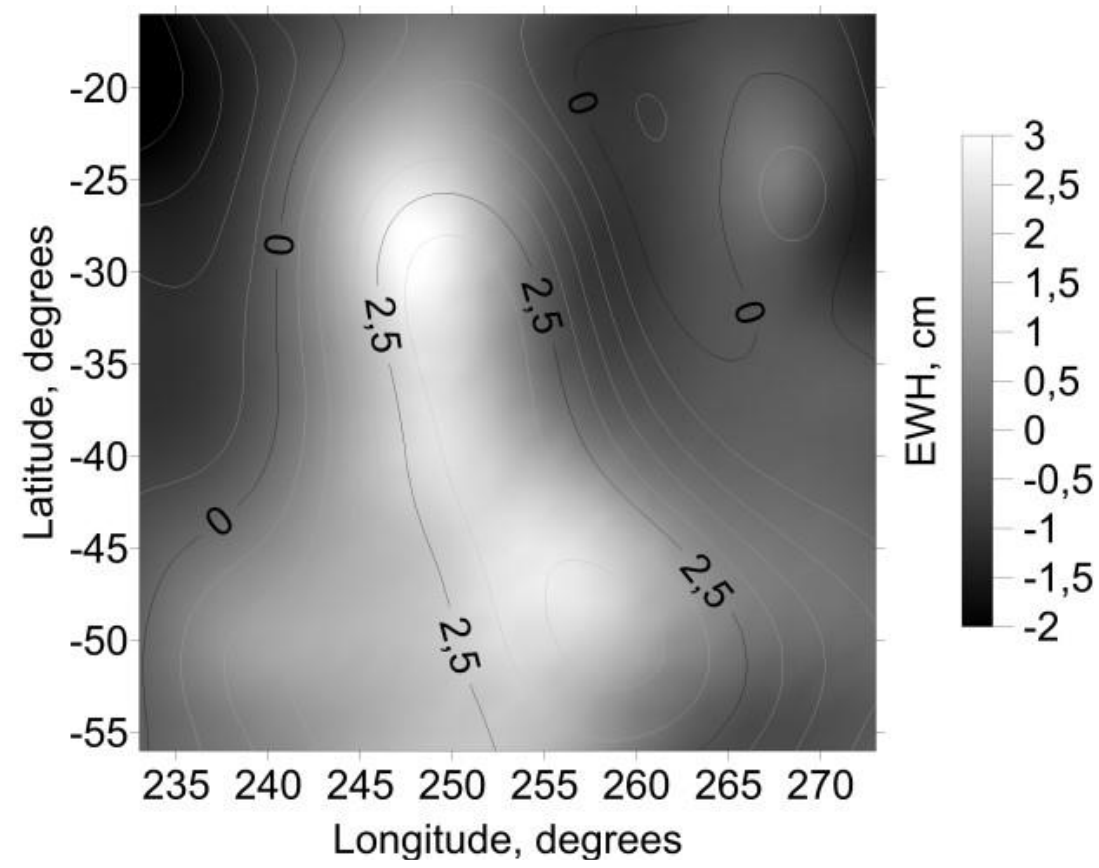

Figure 2. Digital map in isolines of the Chilean earthquake for February 2010 


\section{STATISTICAL ANALYSIS OF GRAVITATIONAL ANOMALIES BY THE METHOD OF MAXIMUM ENTROPY}

For the analysis, it was decided to use the histogram method [6], which is used to determine the probability distribution function for gravitational anomalies. It is created taking into account the redistribution of mass on Earth, which characterizes the current state monthly, in accordance with the registration data. The simplest (model) probability function $\mathrm{W}(\mathrm{h})$ (h is the amplitude of the gravitational anomaly in EWH values) can be determined using a histogram over the entire GRACE satellites observation period.

The obtained histogram of the equivalent water column levels data and its approximation by the Lorentz function with a half-width of $0.92 \mathrm{~cm} \mathrm{EWH}$ is shown. It should be noted that if the probability distribution function is introduced (theoretical Lorentz or selective-experimental histogram) for the value of EWH, then we can introduce an unusual measure of the specific distribution of gravitational field anomalies during the time period (month) when earthquakes occurred. As a measure of the unusual distribution of anomalies near the earthquake epicenter, it is reasonable to use the function of the mean monthly information entropy [7].

Since the occurrence of a strong earthquake is a rare event the problem arises to assess whether something unusual was in a state of gravitational field during this period of time. Also we should take into account the influence of the geometric characteristics of the earthquake preparation zone on the features of the local gravitational anomaly. We will judge the unusualness by the average entropy, which is calculated for a certain neighborhood of the earthquake epicenter and in general on Earth.

Entropy is calculated from the corresponding histograms of the specified areas. Let us consider in detail the analysis of the data of a catastrophic earthquake with a magnitude of $\mathrm{M}=9.1$ (USGS data), which occurred in the region of Sumatra on 26 December 2004. To estimate the state space of the gravitational field anomalies we calculate the corresponding entropies for the December 2004 histogram over the entire Earth (3.52 Nat) and for neighborhood of the earthquake epicentre $\left( \pm 15^{\circ}\right)$ in the region of Sumatra (entropy is 2.698 Nat).

Fig. 3 shows the histograms of gravitational anomalies for December 2004, as well as the dependence of the information entropy of the histogram for an earthquake on the geometric parameters of the source. For a better perception of the histogram, the data of gravitational anomalies are shown in step-histogram and the bar-histogram of the neighborhood $\left( \pm 15^{\circ}\right.$ latitude and longitude) from the epicenter of the earthquake (amplitudes of bars are multiplied by 10$)$.

Comparing the obtained values of the information entropy, we see that the values of the average (global) entropy over the entire observation period (3.66 Nat) are close to the entropy for December 2004 (3.52 Nat). It should be noted that for the focal zone in the area of Sumatra Island the values of entropy (locally) are less $(2.71 \pm 0.02 \mathrm{Nat})$.

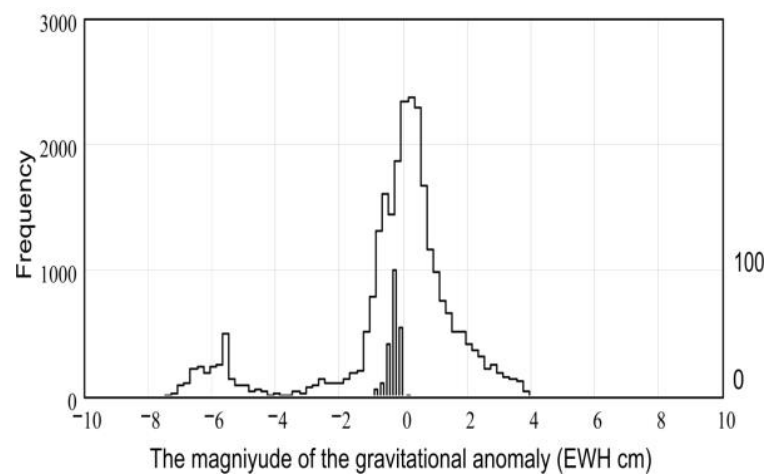

a

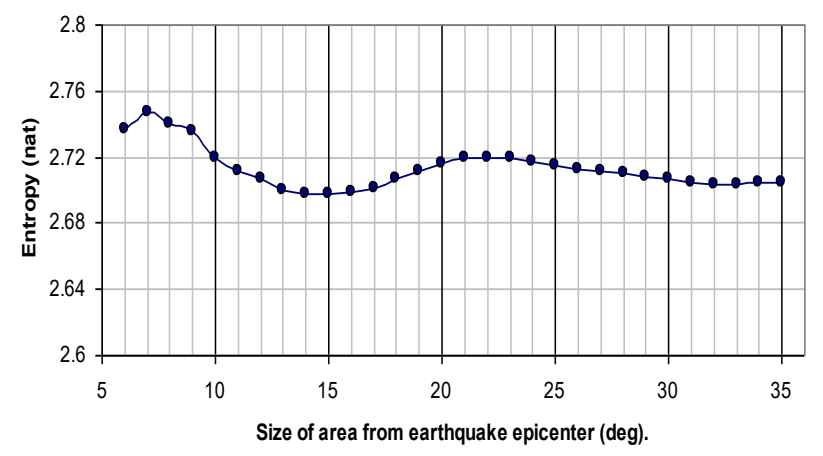

b

Figure 3. (a) Gravitational anomalies histograms for December 2004. (b) Information entropy values depending on the size of the area

Statistical processing of the global variability of the gravitational field anomalies of the Earth according to GRACE (Ocean) for the years 2003-2016 shows that, in general, this variability is random with a differential probability distribution very close to the Cauchy form (Lorentz). This means that there is no significant systematic error in the data. Dominant natural processes are sources of gravity variability over the Earth's ocean. 
The paper shows that there is no sustainable trend, it is excluded by the preprocessing system of registration data GRACE. On the scale of decades there is also no noticeable correlation between the global variability of the Earth's gravitational field and global seismicity. The main component of the gravitational field anomalies of the Earth is associated with the distribution of water masses, i.e. with climate. This means that the strongest earthquakes occur as a result of quasi-local processes. The development of local methods is necessary to predict dangerous earthquakes.

\section{CONCLUSIONS}

The work on the basis of digital and statistical processing of satellite data GRACE solved the problem of description and comparative analysis of the features of gravitational anomalies of seismically active focal zones and the Earth as a whole. The analysis of the focal zone of all the strongest underwater earthquakes in the period from 2003 to 2016 has been performed. For them, digital maps of the distribution of the studied parameter EWH were constructed in the period several months before the main seismic event and several months after it. This information allows us to formulate the features of changes in the studied parameter EWH in the specified periods of increased seismic activity. Digital processing of these data allowed us to select data arrays and target months for detailed statistical analysis.

As a result, we obtained a statistical description of the space of possible states of the anomalies of the gravitational field of the Earth as a whole and features of local gravitational anomalies over the focal zones of the underwater earthquakes of the greatest in the period from 2003 to 2016. Statistical description was carried out on the basis of histogram method in combination with the maximum entropy method.

The following estimates were obtained: for each focal zone, a comparison was made of its state and the state of the Earth as a whole during the period of the studied seismic catastrophe. It is shown that geodynamic zones differ from each other, which is reflected in the character of the constructed histograms and the characteristics of the distribution of entropy depending on the size of the corresponding focal zone.

There are relatively homogeneous regions: seismically active areas (Peru-Chile coast, Sumatra region, Pacific coast Japan and Kuril Islands region). At the same time the features of the state of an ultra-deep earthquake in the Sea of Okhotsk differ significantly in their statistical characteristics from subduction submarine earthquakes.

Additionally, it was revealed that part of the local histograms of the strongest earthquakes are located near zero of the histogram of the Earth as a whole, which corresponds to the most probable state. The histograms of other earthquakes are located in the tails of the basis of the histogram, which, apparently, reflects the geodynamic features of the focal zones. An analysis of the distribution of entropy also shows their uniform monotony for the corresponding geodynamic regions. At the same time, the deep earthquake in the Sea of Okhotsk differs significantly in these statistical indicators.

\section{REFERENCES}

[1] Flechtner, F., Sneeuw, N. and Schuh, W. D., "Observation of the System Earth from Space - CHAMP, GRACE, GOCE and Future Missions," GEOTECHNOLOGIEN. Science Report. Advanced Technologies in Earth Sciences 20 (2014).

[2] NASA, ftp://podaac-ftp.jpl.nasa.gov /allData/tellus/L3/ocean_mass/RL05/ascii/ (12 February 2019).

[3] Wouters, B., Bonin, J. A., Chambers, D. P., Riva, R. E. M., Sasgen, I. and Wahr, J. "GRACE, time-varying gravity, Earth system dynamics and climate change," Review Article Rep. Prog. Phys. 77, 41 pp. (2014).

[4] Wahr, J., Molenaar, M. and Bryan, F., "Time variability of the Earth's gravity field: Hydrological and oceanic effects and their possible detection using GRACE," J. Geophys. Res. 103 (B12), 30205-30229 (1998).

[5] USGS, https://earthquake.usgs.gov /earthquakes/map (14 February 2019).

[6] Anderson, T. W., [An Introduction to Multivariate Statistical Analysis], John Wiley \& Sons, New York, 374 pp. (1960).

[7] Shannon, C. E., "A Mathematical Theory of Communication", Bell System Technical Journal 27(3), 379-419 (2014). 